\title{
Comparaison du fonctionnement du compliment en français et en arabe tunisien
}

\author{
Saloua Zamouri ${ }^{\mathrm{a}}$ \\ Faculté des Sciences Humaines et Sociales, Laboratoire Intersignes, Boulevard du 9 avril \\ 1938, Tunis.
}

\begin{abstract}
Résumé. On pourrait regrouper globalement les recherches contrastives concernant les actes de langage autour de deux grandes tendances qui donnent lieu à un véritable débat : celles qui prônent l'existence d'universaux dans le fonctionnement des actes de langage, et celles qui défendent l'existence de variations culturelles plus ou moins profondes pouvant affecter tous les niveaux de leur fonctionnement. En fait, il semble difficile de trancher sur la part universelle et la part culturellement spécifique des comportements langagiers car il existe dans le fonctionnement des actes de langage à la fois des ressemblances et des différences. Si certaines études contrastives seront attirées par la ressemblance, d'autres mettront surtout l'accent sur la différence. Cette dernière tendance, sans nier l'existence de caractéristiques universelles, a ainsi montré d'une part que des variations profondes affectent le fonctionnement des actes de langage, et d'autre part que ces variations sont pour une large part, étroitement liées à des facteurs culturels. C'est de cette tendance que relève notre approche. Dans la présente communication nous montrerons à travers la comparaison du fonctionnement du 'compliment' dans les interactions françaises et tunisiennes, que cet acte de langage laisse apparaitre de nombreuses variations qui, de notre point de vue, sont révélatrices du système de valeurs et de représentations socio-culturelles des langues-cultures observées. Nous aborderons dans cette étude contrastive le mode de réalisation du compliment, ses conditions de réussite et ses conditions d'emploi pour ensuite aboutir à une comparaison de la valeur socio-cultuelle de cet acte de langage.
\end{abstract}

\begin{abstract}
A cross-cultural comparison of the speech act of compliment in French and Tunisian Arabic. One may group globally the contrastive researches concerning the speech acts around two big tendencies which give rise to a real debate : those who advocate the existence of universals in the functioning of the speech acts, and those who defend the existence of more or less deep cultural variations which can affect all the levels of their functioning. In fact, it seems difficult to separate the universal part from the culturally specific part of the language behaviors for there are in the
\end{abstract}

\footnotetext{
${ }^{a}$ Auteur de correspondance : salwazamouri@gmail.com
} 


\begin{abstract}
functioning of speech acts similarities and at the same time differences. If some contrastive studies are attracted by the similarities, others focus on differences. This tendency, without denying the existence of universal characteristics, has showed on the one hand that deep variations affect the functioning of the speech acts and, on the other hand, that they are largely closely linked to the cultural factors. Our approach belongs to this tendency. In this presentation we will show through the comparison of the functioning of the compliment in French and Tunisian interactions, that this speech act reveals numerous variations which -from our point of vieware revealing of the systems of values and the sociocultural representations of language/cultures that are observed. In this contrastive study we will deal with the mode of realization of the compliment, its conditions of success and its conditions of use, in order to achieve a comparison of the socio-cultural value of this speech act.
\end{abstract}

La présente communication est une réflexion qui s'inscrit dans le cadre de notre travail de thèse, en cours de préparation à l'université de Tunis, dans lequel nous nous proposons de comparer le fonctionnement de certains actes de langage entre le français et l'arabe tunisien; en vue de dégager et d'analyser les variations liées à des spécificités linguistiques et culturelles des langues-cultures étudiées. C'est sur le fonctionnement du compliment que portera la présente communication. En nous appuyant sur un double corpus authentique constitué d'une série de conversations familières françaises et tunisiennes, se déroulant dans les deux cas au cours de visites, nous nous proposons ici de mettre en parallèle le fonctionnement du compliment tel qu'il est attesté dans le corpus. La comparaison portera sur le mode de réalisation et la réaction au compliment, ses conditions de réussite et ses conditions d'emploi. Elle aboutira enfin à des conclusions sur les variations culturelles qui affectent sa valeur socio-relationnelle. Mais avant d'aborder l'analyse proprement dite, nous présenterons dans un premier temps, le cadre théorique et méthodologique dans lequel s'inscrit cette étude.

\title{
1 Cadre théorique et méthodologique
}

Deux courants de recherches servent de cadre théorique et méthodologique à notre travail. Celui-ci s'inscrit d'une part dans le vaste champ de la pragmatique contrastive, et d'autre part dans celui de l'analyse des interactions. Les travaux qui relèvent de la pragmatique contrastive - appelée dans la littérature anglophones Cross Cultural Pragmatics (BlumKulka \& al. 1989 [3] et Wierzbika 1991[32, 33]) ou Contrastive Pragmatics (Oleksy (éd). 1989) [20]- ont globalement pour objectif de comparer des pratiques communicatives attestées dans deux ou plusieurs sociétés et de décrire toutes les variations liées à des spécificités culturelles. Mais à travers la description, ces travaux visent aussi, et peut être surtout, à aboutir à certaines généralisations concernant les sociétés en question; en vue d'expliciter ce que Gumperz (1982, [9]) appelle la «logique culturelle» («cultural logic»). Cette orientation de la pragmatique a suscité chez nous des questionnements sur la nature universelle vs culturelle de nos comportements et nous a concrètement orientée vers une réflexion sur la possibilité de dégager, à partir d'une comparaison de pratiques communicatives, un certain «profil communicatif» des langues-cultures observées : le français et l'arabe dialectal tunisien. Cependant, rapidement nous nous sommes trouvée confrontée aux difficultés mais aussi au caractère risqué d'un tel projet- qu'il s'agisse de dégager un profil communicatif ou même de grandes tendances générales structurées (Kerbrat-Orecchioni, 1994 [14] ) - à partir uniquement d'un seul type de corpus. Un tel projet nécessite en effet une étude étendue qui ferait appel à des données variées, collectées 
dans différentes situations. Dans notre projet de recherche, dans lequel s'inscrit cette contribution, nous avons donc choisi de délimiter le champ de la comparaison à un type particulier de pratique communicative (certains actes de langage). Les tentatives de généralisation qui en seront issues resteront donc relatives et partielles.

Notre travail relève aussi de l'analyse des interactions. D'abord par son objet ; car si notre étude se focalise sur les actes de langage (et plus précisément ici sur le cas du compliment), ceux-ci sont décrits dans leur contexte d'utilisation: celui d'interactions verbales authentiques. Cependant, à la différence de certains autres travaux, il ne s'agit pas dans cette étude d'une analyse comparée de types d'interactions mais d'une analyse comparée de «(...) ressources linguistiques mises en œuvre par les participants dans leurs échanges» (Traverso, 2006[31]). La méthodologie que nous adoptons est aussi celle de l'analyse des interactions : notre étude s'appuie en effet sur un corpus de conversations authentiques se déroulant entre locuteurs engagés dans des situations de rencontres sociales réelles; et notre démarche est essentiellement inductive et qualitative.

La richesse et la complexité d'une étude contrastive des actes de langage nous ont amenée à adopter une approche éclectique qui associe des outils descriptifs issus de différentes disciplines: théorie des actes de langage, théorie du face-work, pragmatique contrastive, analyse des interactions et autres approches interactionnelles, linguistique.... Car nous pensons, comme le soutient Kerbrat-Orecchionni (2005: 21, [17]), qu'il est «plus intéressant et rentable de concilier ce qui est conciliable, et de voir le parti que l'on peut tirer du croisement de propositions provenant de paradigmes différents».

\subsection{Présentation du corpus}

Le point de départ de notre réflexion sur le compliment a été l'ouvrage de Traverso intitulé la conversation familière (1996, [27]), dans lequel l'auteure décrypte différents aspects du fonctionnement de conversations familières se déroulant au cours de visites. Parmi les pratiques communicatives étudiées par l'auteure, figure le compliment. Etant une native de l'arabe, pour qui le français constitue une langue seconde, nous avons tout de suite été frappée par le contraste qu'il peut y avoir dans les valeurs socio-relationnelles et interactionnelles attachées à cette pratique dans les deux langues/cultures en question. Nous avons donc voulu mettre en parallèle le fonctionnement du compliment, en vue de relever les éventuelles variations liées à des spécificités pragmatiques et culturelles.

Dans son étude ${ }^{1}$, l'auteure s'est appuyée sur un corpus constitué de « 25 conversations enregistrées au cours de visites entre 1986 et 1992, à Lyon» (Ibid.: 23)2 . C'est ce corpus que nous utilisons comme terme de la comparaison. Pour cela, nous avons entrepris de constituer un deuxième corpus équivalent (cette question de l'équivalence sera discutée en 1.2.) en arabe dialectal tunisien.

Dans cette étude nous nous appuyons donc sur un double corpus :

- un corpus français emprunté à Traverso (1993,[26] 1996[27]) sur lequel nous fondons les éléments descriptifs relatifs au fonctionnement du compliment en français.

- un corpus en arabe dialectal tunisien constitué de 21 conversations se déroulant dans le cadre de visites rendues aux uns et autres, en Tunisie (dans trois régions différentes du pays) ${ }^{3}$.Nous avons effectué nous- même les enregistrements (audio), dans tous les cas à l'insu des participants; car dans toutes ces visites nous étions présente (ce qui a constitué un avantage lors de sa transcription). La totalité des enregistrements représente une vingtaine d'heures d'enregistrement (mais certains passages n'étaient pas exploitables pour nous car ne répondant pas à notre objectif de recherche). Nous avons ensuite procédé à sa transcription, puis à sa traduction. Pour les passages soumis à l'analyse, deux niveaux de traduction se sont révélés nécessaires: un premier niveau de traduction littérale qui a l'avantage de rendre compte de spécificités linguistiques, sémantiques ou interactionnelles, et un deuxième niveau de traduction adaptée ${ }^{4}$ plus lisible pour un locuteur francophone. 
Pour ce qui est des échantillons étudiés ici, nous avons dû transcrire les passages du corpus arabe en alphabet latin et non pas en API (comme c'est le cas généralement). La traduction sera, en fonction du but recherché, soit littérale soit adaptée.

Cependant, dans certains cas lorsque le corpus de base ne fournit pas suffisamment de données ou d'occurrences nécessaires à l'analyse, nous avons dû faire appel à d'autres sources : exemples pris sur le vif ou rapportés, données fournies par différents auteurs. Ces sources seront signalées en tant que telles.

\title{
1.2 Comparabilité et représentativité ${ }^{5}$
}

La fiabilité d'une analyse contrastive, on le sait, dépend en premier lieu de la comparabilité des situations choisies. En effet, pour être comparables, les situations choisies doivent se définir de façon similaire, ou tout au moins suffisamment proche, pour considérer que l'on peut, sur cette base, procéder à une comparaison (Traverso, 2006 [30]). Pour assurer cette comparabilité nous avons tenté de constituer un corpus équivalent à celui du français, reposant sur des similitudes concernant le type d'interaction et la situation (des conversations de visites), la nature de la relation (amicale et/ou familière), l'âge (entre 30 et 70 ans) et le sexe des participants (hommes et femmes).

Cette exigence n'est cependant pas des plus facile et évidente à réaliser ; les situations strictement identiques n'existant pas d'un pays à l'autre. Car même lorsque nous nous trouvons face à des situations en apparence identiques, comme c'est le cas pour les visites, il se trouve que dans ce cas, leur déroulement, du fait des variations culturelles et socioéconomiques, est si spécifique qu'il n'est pas possible de parler d'activités identiques ; elles seraient, comme l'explique Traverso $(2000 \mathrm{a}: 34,[28])$ tout au plus similaires :

\begin{abstract}
Se rendre chez quelqu'un de sa connaissance pour passer un certain temps en sa compagnie est sans doute une activité répandue de par le monde. Mais les éléments qui permettent de la comprendre (son inscription dans un système d'échanges, son rôle social, son caractère plus ou moins obligatoire, sa relation au temps, etc.) conduisent à se demander si au fond la visite est bien une situation / activité similaire, en France et en Syrie par exemple.
\end{abstract}

Et justement, en comparant le déroulement de la visite en France et en Tunisie, nous avons relevé des divergences, lesquelles portent en particulier sur son rôle social, sur les règles qui régissent les visites prévues et les visites imprévues, ou encore sur les rituels déployés.

L'exigence de comparabilité concerne aussi le type d'activité. Les interactions concernées par cette étude sont, dans les deux corpus, des conversations ordinaires se déroulant entre familiers dans le cadre des visites. Il s'agit de «parole qui se manifeste quand un petit nombre de participants se rassemblent et s'installent dans ce qu'ils perçoivent comme étant une courte période coupée des (ou parallèles aux) tâches matérielles ; un moment de loisir ressenti comme une fin en soi, durant lequel chacun se voit accorder le droit de parler aussi bien que d'écouter, sans programme déterminé» (Goffman, $1974: 20$ [8]). Le français utilise le terme « conversation » pour désigner ce type d'échange ; en arabe tunisien on y renvoie en employant le terme « h'dith » qui possède lui, un emploi plus général recouvrant différents types d'échanges verbaux. Pour désigner la conversation, les locuteurs tunisiens recourent aussi à une périphrase : « h'kina » (qui peut se traduire par « on a échangé des paroles ». Mais même s'il n'y a pas d'équivalent lexical identique au mot « conversation », il n'en reste pas moins que ce type d'activité est bel et bien attesté dans la société tunisienne (et semble d'ailleurs universel). Cependant, le fait que la même activité soit attestée ne signifie pas pour autant, rappelons-le, que cette activité soit similaire. Dès le moment où une même activité est réalisée dans des contextes culturels différents, nous pourrions en effet supposer qu'elle puisse présenter des variations. 
Enfin, la comparabilité touche aussi, et surtout pour l'étude qui nous occupe ici, le fait soumis à la comparaison : le compliment. Nous nous proposons en effet de comparer un acte de langage donné, que nous désignons en utilisant une expression française : «le compliment» comme si cela allait de soi que le même acte existe aussi en arabe tunisien. Or, on le sait, même lorsque les actes de langage sont en apparence identiques, leurs règles pragmatiques et interactionnelles, leurs conditions de réalisations et autres divergences sont « parfois si différentes d'une langue à l'autre qu'il n'est pas possible d'établir clairement qu'il s'agit du même acte » (Kerbrat-Orecchioni, 2001 :169 [16]). Cette question est justement l'objet de cette étude.

Pour pouvoir procéder à la comparaison nous nous appuierons donc sur des propriétés générales communes aux deux corpus observés (en ce qui concerne la situation, le type d'activité et l'acte de langage étudié). Propriétés qui transcendent en quelque sorte les divergences en une définition générale valable pour les deux langues étudiées, un tertium comparationis sur lequel fonder l'analyse.

\subsection{Représentativité des corpus}

Le travail contrastif procède nécessairement à des généralisations. Or, s'il est indéniable que l'on peut voir dans la langue un reflet de la culture, on peut aussi se poser la question de savoir si les comportements langagiers et communicatifs dégagés à partir d'un corpus donné sont représentatifs de cette culture et s'ils permettent un niveau de généralisation satisfaisant. En d'autres termes, et en l'occurrence pour le cas de cette étude, le fonctionnement du compliment tel qu'il est attesté dans le corpus est-il suffisamment représentatif pour nous permettre de le généraliser à l'ensemble des Tunisiens ou des Français.

Pour prétendre aboutir à des généralisations, une recherche doit-elle pour cela s'appuyer sur un corpus très large prenant en compte toutes les variations intra-culturelles ? Selon Béal (2000:20 [2]) en effet, «L'idéal serait un échantillon large dans lequel se fondraient toutes les micro-cultures qui peuvent être liées à l'âge, au sexe ou à la classe sociale des participants ». Cependant, il est aussi possible, comme le souligne l'auteure elle-même, d'aboutir à des généralisations relatives à partir d'une analyse qualitative d'échantillons restreints.

Cependant, on peut tout de même faire ressortir la variable 'interculturelle' à partir d'échantillons étroits, mais dans ce cas-là, il faut que ceux-ci soient par ailleurs semblables : si toutes les autres variables sont les mêmes (niveau d'éducation, milieu social, type d'activité...) les différences pourront être attribuées à la différence de culture ; elles seront peut- être un peu grossières, mais fiables. (Ibid. $: 20)$

Nous pensons que, grâce à un corpus le plus équivalent possible, à une bonne connaissance des cultures observées et au besoin, au recours à des discussions avec des locuteurs natifs, il est possible pour l'analyste de dégager des tendances générales. Ceci, tout en veillant à tenir compte de la relativité des données.

\subsection{Problèmes du métalangage}

Comment appréhender l'étude d'une pratique communicative ancrée dans une culture donnée au travers du prisme des termes descriptifs d'une autre langue ? Etant donné que nous nous proposons de comparer le fonctionnement d'un acte de langage dans deux langues différentes, tout en choisissant comme langue de description une langue particulière, en l'occurrence le français, nous encourrons ici le risque de "plaquer » en quelque sorte des concepts et une terminologie propres au français à un corpus non français. 
Cette problématique, bien connue en pragmatique contrastive, ne peut pas être complètement évacuées. En revanche, ce problème de métalangage et de catégorisation des faits peut être réduit si l'analyse remplit certaines conditions ; entre autres :

- sans aller jusqu'à utiliser le métalangage («primitifs sémantiques») établi par Wierzbika (1985[33]et 1991[34]), lequel présente l'inconvénient d'être trop complexe, il est possible de s'en inspirer pour adopter une terminologie la plus neutre et générale possible, englobant les deux langues.

- se dégager de sa grille de lecture culturelle pour atteindre une position neutre et objective. - analyser de l'intérieur les corpus à étudier avant de procéder à la comparaison. KerbratOrecchioni (1994 [14],2005 : 288-295[17]) par exemple propose, pour minimiser les risques, de décrire de façon extrêmement précise la culture représentée dans un corpus donné.

Quoi qu'il en soit, ces difficultés existent, elles restent inhérentes à toute comparaison interculturelle. A l'analyste d'en prendre conscience et d'exiger de lui-même une extrême vigilance et une grande méticulosité dans l'interprétation des résultats.

\section{DESCRIPTION}

\subsection{Identification du compliment ${ }^{6}$}

Pour une description contrastive comme la nôtre, catégoriser un énoncé comme étant 'un compliment' est une tâche délicate. En effet, le premier problème qui se pose est la question de l'équivalence de cet acte de langage dans les deux langues étudiées : ce que nous aurons sélectionné comme acte complimenteur en français à partir duquel se fera la comparaison, a-t-il son équivalent en arabe tunisien ? Lorsque l'on sait que dans la langue arabe (classique ou dialectale) l'équivalent lexical de «compliment » et « complimenter » n'existent pas et qu'à la place on trouve des termes qui renvoient à l'idée générale de «louange », ou « éloge » dont le compliment ne constitue qu'un cas particulier (le compliment c'est une louange qui porte sur le destinataire), on imagine les problèmes et les interrogations que posent notre description contrastive de l'acte de langage en question.

\section{Le compliment en français}

A la suite de Kerbrat-Orecchioni (1994 : 202[17]), nous définissons le compliment comme : $1-\ll$ Toute assertion évaluative positive,

2- portant sur une qualité ou une propriété de l'allocutaire A (un compliment, c'est une louange adressée à la personne « concernée»),

3- ou bien encore, sur une qualité ou propriété d'une personne plus ou moins étroitement liée à $\mathrm{A}(\ldots)^{7} »$

Ainsi, la focalisation d'un énoncé élogieux sur l'interlocuteur est fondamentale dans la définition du compliment en français. Cela l'oppose à la louange, concept très proche: «On peut louer n'importe qui ou n'importe quoi, mais on ne peut complimenter que son interlocuteur- le verbe « louer » étant en ce sens hyperonyme de «compliment » (KerbratOrecchioni, $1994: 203)$.

\section{Le compliment en arabe tunisien}

En arabe tunisien (comme en arabe classique) cette distinction établie à partir de la nature du destinataire d'un énoncé élogieux ne semble pas pertinente. Ce qui est catégorisé comme compliment en français, ne constitue en définitive pour les Tunisiens, qu'une louange adressée à l'allocutaire, exprimée par les verbes « madaha » ou « chakara» ${ }^{8}$. Mais s'il n'existe pas un terme pour désigner plus spécifiquement les énoncés louangeurs focalisés 
sur l'allocutaire, cela nous autorise-il à dire que le concept de compliment n'existe pas ? En fait, nous pensons que cette absence ne signifie pas que l'acte en question soit inconnu pour cette langue 9 . Le compliment, en tant qu'énoncé élogieux focalisé sur l'allocutaire, est bien attesté même s'il n'est pas décrit par un terme spécifique. Selon Verschueren (1981 [32]) certaines routines conversationnelles sont en quelque sorte 'oubliées' du système d'une langue dans la mesure où ils ne possèdent pas de verbes spécifiques pour les décrire (« forgotten routines ») : «It is the set of non-lexicalized conversational routines that I call forgotten routines $(\ldots) »($ ibid :135).

Lorsque l'on sait en plus, comme nous le montrerons dans ce travail, que le compliment ne constitue pas à proprement parler un acte routinier, comme cela semble être le cas pour le français, on pourrait penser, à la suite de ce qui est avancé par Verschueren (1981: 153) qu'il y aurait là une explication à cet 'oubli' :

\begin{abstract}
(...) the gaps in the lexical frame associated with linguistic action are due to the low cognitive salience of the corresponding acts. This principle, if it is correct, proves that there is a relationship between the lexicon, or the lexicalization process, and habits of conceptualization. Hence, this chapter confirms our belief that studying and comparing the verbials available in different natural languages for the description of linguistic action may yield insights into people's language-specific and culture-specific conceptualization and experience of linguistic behavior and, therefore, into the nature of linguistic behavior itself".
\end{abstract}

Mais ce que nous avançons là ne constitue pour l'instant que des interrogations qui nécessitent une réflexion plus approfondie. Quoi qu'il en soit, il est clair qu'il y a entre le français et l'arabe une différence de catégorisation. Tandis qu'en français, dans la catégorie des énoncés élogieux, les énoncés qui sont focalisés sur le destinataire sont isolés des autres et décrits avec un verbe spécifique; en arabe, la catégorie particulière des «-énoncésélogieux -focalisés - sur- le- destinataire » ne sont pas considérés comme une classe à part mais englobés dans la catégorie plus large de la louange et décrits en tant que tel.

\title{
2.2 La réalisation du compliment
}

\section{En français}

Dans sa description de l'échange complimenteur dans les conversations familières françaises, Traverso (1996 : 93-94[27]) montre à partir de son corpus de référence que le compliment se réalise en français selon un certain schéma stéréotypé. L'auteure dégage principalement quatre structures entre lesquelles se répartissent la majorité des compliments :

Forme1

$\mathrm{Tu}+\hat{e}$ tre + axiologique positif (+spécification)

Selon l'auteure cette forme implique totalement la personne complimentée puisque le compliment est focalisé sur la personne (tu)

Ex : « Ouais, t'es toute belle »

Forme2

$\begin{array}{llll}\text { Ce } & X & \text { te va } & \text { (très) } \\ \text { Ton } & & \text { (parfaitement) } & \text { bien } \\ \text { Le } & & \text { (super) }\end{array}$


Les compliments de ce type étant focalisés non pas sur la personne elle-même mais sur un élément de son apparence :

Ex : « ta coupe te va super bien »

Forme3

Tuas un + axiologique $+\quad X$

Cette forme concerne les compliments focalisés sur un objet possédé par l'allocutaire. Mais contrairement à la forme2, l'allocutaire n'est ici mentionné que dans sa relation de possession à l'objet complimenté ${ }^{10}$.

Ex : «Tu as un joli pull »

Forme 4

Elle prend la forme d'un énoncé de type «J'aime bien (ton X) ».

Mais, selon l'auteure, cette forme " semble n'être pas toujours considérée comme un compliment, mais plutôt comme l'expression d'une opinion ou d'un sentiment » (Ibid. : 94)

Nous ne chercherons pas ici à entrer en détail dans la description de ces formes. En revanche, ce qui nous semble important à relever est le fait que, quelle que soit la forme choisie, le compliment comporte toujours une évaluation positive explicite portant (directement ou indirectement) sur l'allocutaire; le complimenteur se posant comme la source évaluative de l'énoncé. D'autre part, d'après l'observation des réalisations du compliment dans le corpus, et d'après les différents travaux consacrés à cet acte (KerbratOrecchioni, 1994[14]; Traverso, 1996[27] entre autres) il ressort qu'en français, l'évaluation positive s'exprime le plus souvent par des termes intrinsèquement axiologiques ${ }^{11}$, essentiellement des adjectifs.

\section{En arabe tunisien}

Comme pour le français, le compliment (dans sa réalisation canonique explicite) s'exprime en arabe tunisien par des énoncés assertifs comportant une évaluation positive focalisée de manière directe ou indirecte sur l'allocutaire. Ces énoncés se réalisent sous des formes figées qui varient peu aussi bien au niveau de leurs structures syntaxiques qu'à celui du choix du lexique employé. Ils comportent aussi dans leur majorité un axiologique positif et un mot spécifiant l'objet ou la personne complimenté :

Ex.: « hlouwa a-rouba » (litt. jolie la robe)

«mizyana bint-ik » (litt.belle ta fille)

Des morphèmes exclamatifs sont parfois employés pour intensifier le jugement admiratif du complimenteur.

Ainsi nous pouvons constater que sur certains points les deux types de compliments, français et arabe, sont comparables. Cependant, un trait important caractérise ce dernier. En effet, à la différence du français, l'évaluation positive contenue dans le compliment s'accompagne presque toujours d'une ou de plusieurs formules lexicalisées à valeur conjuratoire lesquelles peuvent soit précéder soit suivre l'évaluation positive. La forme canonique la plus attendue du compliment prend ainsi la forme :

- Evaluation positive (focalisé sur l'interlocuteur un objet possédé)

Ex. « hlouwa a-rouba

(litt. Jolie la robe
+ formule $(s)$ conjuratoire $(s)$

+ tbark-allah»

+ 'Que Dieu bénisse') 
- Formule $(s)$ conjuratoire $(s)+\quad$ évaluation positive (focalisée sur l'interlocuteur ou sur un objet possédé)

Ex. «tbark-allah + dar-ik kbira»

(litt. 'Que Dieu bénisse' $\quad+$ ta maison est grande)

Ces formules conjuratoires, si elles ne sont pas obligatoires, sont en tout cas attendues, plus ou moins fortement selon le contexte et le thème du compliment (voir 2.3). Au-delà d'une simple différence dans le mode de réalisation c'est la valeur même du compliment qui est ici concernée. En effet, pour les Tunisiens, un compliment réussi doit certes faire plaisir au destinataire en le flattant mais il doit aussi et en même temps le protéger contre le jugement admiratif porté sur lui. Le protéger, car le jugement admiratif est considéré comme l'expression (volontaire ou non) de l'envie et peut à cause de cela, entrainer le 'mauvais œil'.

Les formules employées font partie d'un système plus large et complexe qui, en arabe, couvre un espace pragmatique important au sein des différentes situations interactionnelles ; pouvant apparaître dans toutes sortes d'occasions et servir à accomplir différents buts illocutoires. La plupart ont une origine religieuse : ce sont des sortes d'invocations. Mais les formules religieuses en côtoient d'autres qui sont issues de la tradition populaire. Les formules énoncées dans le contexte du compliment (et de la louange de manière plus générale) ont, elles, une fonction particulière de conjuration. Leur action supposée et voulue et d'invoquer la protection divine contre les effets potentiels du 'mauvais œil' pouvant toucher la personne ou l'objet admirés. Plusieurs formules sont attestées, mais les plus fréquentes dans notre corpus sont «tbark-allah » et «msali a-nibi ».

La formule « tbark-allah » (Litt. 'Que Dieu bénisse') comporte un dérivé verbal de l'arabe littéraire «baraka » qui, en gros, peut se traduire par « Force bénéfique, d'origine divine, qui provoque la surabondance dans le domaine physique, la prospérité et la félicité dans l'ordre psychique ». La «baraka » est une des valeurs les plus recherchées et les plus prisées dans la culture arabo-islamique. Quel que soit le contexte et le dérivé employé, son explicitation est toujours investie d'une valeur conjuratoire très forte. Associée à l'expression de l'admiration, qu'il s'agisse ou non de compliment, elle a une fonction prophylactique. On considère en effet, que sa seule énonciation est susceptible d'annihiler l'effet néfaste du 'mauvais œil' contenu potentiellement dans l'admiration et donc de protéger la ou les personnes et/ou les objets admirées.

La formule « msalli a-nibi » (Litt. 'prie (injonctif) sur le prophète') est un dérivé dialectal de la formule rituelle « allah-oma sali ala a-nabi» (Litt. 'Notre Allah prie sur le prophète') laquelle est littéralement une formule de prière sur le prophète, et est considérée chez les Musulmans comme faisant partie des invocations à travers lesquelles on se rapproche du Seigneur ${ }^{12}$ (Allah). Cependant, comme c'est le cas pour la plupart des formules d'invocation qu'on appelle couramment "formules de bénédiction », la valeur religieuse contenue littéralement, est en quelque sorte érodée par l'usage ${ }^{13}$.

Dans notre corpus, ces deux formules sont de loin les plus employées. En voici quelques exemples :

Exemple1 (L1 faisant visiter sa maison à L2) :

L1- hadhi bit noum (ça c'est la chambre à coucher)

L2- behia tbark-allah (elle est bien 'Que Dieu bénisse')

L1- $[\ldots]$

L2- msalli a-nibi kbira w wasâa ('prie sur le prophète' elle est grande et vaste)

Mais très souvent, la formule conjuratoire en vient à elle seule à réaliser le compliment (l'évaluation positive étant sous-entendue) : 
Exemple 2 (L1 raconte à L2 comment ses filles, qui sont présentes à la conversation, l'ont aidée dans une tâche ménagère) :

L1- $[\ldots]$

L2- walahi tbark-allah alihom (Litt. vraiment 'Que Dieu les bénisse ')

En voici un autre exemple :

Exemple3 (L1 regarde la petite fille de son interlocutrice puis, admiratrice, énonce : )

L1- «bint-ik tbark-allah aliha» (Litt. ta fille 'Que Dieu la bénisse')

Dans ces deux derniers exemples, il s'agit de compliment « indirect » qui concerne la (les) fille(s) de l'interlocutrice, donc une personne à laquelle l'interlocutrice est liée. Mais, dans l'exemple 2 il s'agit aussi d'un compliment indirectement adressé à L3 (une jeunefille) puisque cette dernière est présente dans l'interaction (mais ne réagit pas au compliment).

De la même manière, certaines formules figées en sont venues à exprimer de manière spécifique les compliments focalisés sur un élément nouveau. Les plus employées dans ce contexte sont «sahha » ('santé) et « mabrouk » ('bénédiction') :

Exemple4 (L1 remarque la nouvelle coupe de cheveux de son amie ) :

L1- ah sahha qasit chaâr-ik ? (ah "santé" tu t'es coupée les cheveux?) L2- y-aât-ik isahha [...] (Litt. Qu'Il te donne la santé) ${ }^{14}$

En fait tout se passe comme si, étant toujours associées à l'expression de la louange, ces formules ont fini par l'absorber. La tournure elliptique « tbark-allah + particule référent au destinataire », la plus utilisée dans ce cas, concerne plus particulièrement les compliments globaux focalisés sur la personne. Comme on peut le voir dans les exemples 2 et 3, ces formules peuvent occuper, au niveau syntaxique, la place d'un prédicat.

Mais les formules conjuratoires peuvent en fait accompagner non seulement le compliment mais aussi, de manière plus générale, toutes formes d'expressions d'admiration (éloge, admiration concernant un tiers ou un objet). Dans ce cas, elle peut être énoncée soit par le locuteur lui-même soit par l'allocutaire :

Exemple5 (L2 décrit en termes élogieux la maison de son fils)

1 L2- wa kba::r tbark-allah (et elles sont grandes[les pièces] 'Que Dieu bénisse')

2 L2- $[\ldots]$

3 L1- tbark-allah kbira barcha ( Litt. 'Que Dieu bénisse' elle [la maison] est très grande)

4 L2- kbira barcha.. zouz byout banouat.. tbark-allah wa tlatha byout raha machallah [...]

5 (Litt. Elle est très grande...deux salles de bains "Que Dieu bénisse" et trois toilettes 6'Que Dieu le veuille' (cette dernière formule étant une variante de 'tbark-allah' et joue le 7 même rôle) $[\ldots]$

8 L1- msalli a-nibi (Litt. 'Prie sur le prophète')

Comme dans l'exemple qui précède, l'énonciation de la formule «msalli a-nibi » (1.8) constitue à elle seule un compliment (indirect dans cet exemple puisque le contenu concerne L3, le fils de l'interlocutrice).

\subsection{La réaction au compliment}

L'énoncé complimenteur, que ce soit en français ou en arabe, est en même temps une assertion évaluative et une sorte de « cadeau verbal » (Kerbrat-Orecchioni, 1994 : 228 [14]) destiné à faire plaisir à son destinataire. Celui-ci, comme réaction à ce compliment a donc 
la possibilité d'enchaîner soit sur l'assertion, soit sur le «cadeau verbal» (soit sur les $\operatorname{deux})^{15}$.

En ce qui concerne le premier cas, Traverso (1996 : 96-106 [27]) dont l'étude nous sert de référence, distingue principalement deux cas de figure pour le français :

1-L'accord sur l'assertion, qui peut être plus ou moins explicite et plus ou moins nuancé:

Ex. A- il est super ton pantalon

$C$ - j'adore il est en coton gratté tu sais

L'auteure montre, à partir des exemples étudiés, que la réaction du bénéficiaire du compliment est en quelque sorte régie par des règles de comportement qui « interdisent de valoriser exagérément sa propre face » (Ibid.: 97). Ces règles poussent ainsi le complimenté à une formulation presque toujours atténuée, (minimisation de l'évaluation, adjonction d'un commentaire négatif...).

2-Le désaccord sur l'assertion:

Ex. L- oh comme c'est joli ta tenue

M- et ben pourtant c'est une vieillerie

Dans le cas d'un désaccord, deux explications nous semble possibles : soit il s'agit encore d'une stratégie de minimisation de l'évaluation positive où on se dévalorise, conformément au principe de «modestie » (Leech, 1983[19] ; Orecchioni, 1994[14] ), soit il s'agit d'un désaccord sur la véracité de l'affirmation (mettant en doute le bien- fondé de l'affirmation).

En arabe tunisien, les deux types de réactions (accord et désaccord) sont attestés et les tentatives de minimisations guidées par la modestie sont courantes. Cependant, en observant le fonctionnement du compliment nous avons aussi relevé des cas où la minimisation se justifie non pas par le souci de modestie, mais plutôt par le besoin d'enlaidir' en quelque sorte l'objet du compliment pour en éloigner le mauvais œil :

Exemple 6 (L1, collègue de L2, découvre avec admiration la nouvelle voiture achetée par cette dernière)

L1- ya:::chrit karhba/ mahla::ha (oh! Tu as acheté une nouvelle voiture! Comme elle est belle !)

L2- ti khashal... ahayka (déjà) tahitli (en panne) (tu parles! elle est déjà tombée en panne !)

On constate ici que le compliment formulé par L1 n'est pas accompagné de la formule conjuratoire, normalement attendue dans ce contexte. Ce qui, probablement, confère au compliment une certaine menace justifiant la réaction de L2.

Le compliment étant, pour l'une et l'autre culture, une sorte de « cadeau verbal » destiné à faire plaisir à son destinataire, l'enchaînement se fait aussi sur cette intention. Dans les différents travaux réalisés sur le compliment en français (Marandin, 1987[20] ; Kerbratorecchioni,1994[14]; Traverso,1996[27], entre autres) il ressort que la réaction normalement attendue et attestée est l'acceptation; le refus étant, lui, très marqué.

En arabe tunisien, le compliment, et toutes autres formes d'éloge ${ }^{16}$, est normalement accompagné d'une formule conjuratoire laquelle garantit en quelque sorte son succès en tant que «cadeau verbal ». La réaction est dans ce cas l'acceptation. Celle-ci se réalise le plus souvent à travers une expression de gratitude qui prend la forme d'une (ou plus) formule(s) de bénédiction :

Exemple7 (L1 regarde la fille de son interlocutrice, puis énonce : )

L1- bint-ik tbark-allah aaliha (Litt. ta fille 'Que Dieu la bénisse', ce qui signifie dans ce contexte « ta fille est jolie »)

L2- y-aich-ek..rabi y-fadhal-ik lawlad (litt.' Qu'Il te fasse vivre'.. 'Que Dieu garde tes enfants') 
L'intervention de L2 comporte deux formules de bénédiction qui ont la valeur d'une expression de gratitude.

Mais il arrive aussi que le compliment (et autres formes d'évaluations positives) ne soit pas accompagné d'une formule conjuratoire. Ce cas n'est pas représenté dans le corpus (lequel nous avons vu fournit peu d'occurrences pour le compliment) mais il est possible et est attesté. En voici un exemple pris sur le vif, dans lequel L1 exprime un certain contentement dû à l'abondance de la pluie:

Exemple8 :

L1- esna mtar barcha... chta kamil w hiya tsob (cette année il y a beaucoup de pluie...il a plu tout l'hiver)

L2- ti qoul msali anibi/ (mais dis 'prie sur le prophète'!)

L3- msali anibi rabi yarhamna ('prie sur le prophète'...'Que Dieu nous accorde sa miséricorde')

Dans cet exemple, la réaction de L2 (un rappel à l'ordre) montre combien l'expression de l'admiration peut être ressentie comme une menace lorsqu'elle n'est pas accompagnée de formule conjuratoire. Menace d'autant plus forte que l'objet de l'admiration (la pluie) dans un pays majoritairement agricole et semi-désertique où la pluie peut manquer, est considéré comme une bénédiction.

Ce type de réaction est aussi attesté dans le compliment, cependant nous avons constaté que la 'sommation' vient en général d'une tierce personne, présente dans l'échange :

Exemple9 (L1 fait des compliments à L2 au sujet de ses enfants. L3 est une parente aux deux interlocuteurs)

L1- wlad-ik il kol najhin fil qraya (tous tes enfants ont réussi dans leurs études)

L2- [absence de réaction verbale]

L3- ay tbark-allah alihom (oui 'Que Dieu les bénisse').

Cependant, il peut arriver aussi que la personne concernée- si elle a suffisamment de caractère- se charge elle-même de la réparation, parfois sous la forme de plaisanterie (ce type de réaction nous a été rapporté).

Dans d'autres cas, en revanche, le destinataire accepte le compliment (à travers une expression de gratitude en usage), mais alors très souvent cette acceptation n'est qu'apparente car elle cache en réalité une perception désagréable du compliment reçu. En voici l'exemple de deux récits racontés (au cours de deux conversations différentes) :

Récit1 (une locutrice indignée raconte la scène à une autre interlocutrice, en présence de deux autres participantes)

« Tu sais ce qu'il m'a dit? Il m'a dit « Ta mère elle est encore en bonne santé hein ! » tu te rends-compte ? (exclamation générale et indignation). Je cherchais quelque chose à lui dire pour heuh...ou au moins quelque chose à formuler intérieurement...J'aurais dû lui réponde «Sali anibi (prie sur le prophète) »! »

Récit2 (la locutrice est une femme d'environ 70ans)

«J'étais dans un mariage. A un moment je me suis levée, et une femme, une cousine, m'a dit «Mais tu es encore en forme ! ». Dommage, je n'ai pas su réagir, j'aurais dû lui dire «Dis sali anibi (prie sur le prophète) » au moins !». En rentrant chez moi je suis tombée malade. Mon mari m'a dit en plaisantant un peu «Il fallait lui répondre «Tu sais, c'est parce que je mange du poisson ${ }^{17}$ ».

Pour conclure au sujet de la réaction au compliment, nous dirons qu'elle dépend en grande partie d'un certain nombre de facteurs tels que : la nature de la relation qui existe entre le complimenteur et le destinataire du compliment, leur niveau socio-culturel (chez 
des personnes de culture occidentale on retrouve ce que nous pouvons appeler « le compliment à l'occidental » focalisé sur un aspect de l'apparence physique et fonctionnant comme une routine), et la nature de l'objet sur lequel porte le compliment. Car la perception positive ou négative qui se dégage du compliment peut être proportionnelle à la nature de l'objet du compliment. En effet, si certains thèmes sont devenus aujourd'hui anodins : aspect particulier de l'apparence (coupe de cheveux, nouveaux vêtements), d'autres sont considérés comme plus sérieux (avec lesquels on ne plaisante pas) tel que le thème de la pluie évoqué plus haut, ou encore la santé, la progéniture, la beauté physique...Une étude de l'échange complimenteur nécessite donc une description plus poussée qui dépasse le cadre de cette contribution.

\subsection{Conditions de réussite du compliment}

En résumant, nous dirons que tout en étant une assertion, le but illocutoire du compliment est de faire plaisir à son destinataire (en flattant sa face positive). Pour cela, l'énoncé doit satisfaire certaines conditions. Pour le compliment en français nous retiendrons les suivantes, proposées par Kerbrat-Orcchioni (1994 : 257) :

- « condition de sincérité

- conditions concernant le locuteur : que ce dernier apparaisse comme compétent (c'est-àdire relativement exigeant, et suffisamment estimable.) et qu'il soit autorisé à émettre le compliment en question.

- condition sur la pertinence de l'énoncé : le compliment doit être susceptible de faire plaisir à son destinataire en le valorisant et pour ce faire, le contenu de l'énoncé doit être conforme au système de valeurs du destinataire. »

Ces conditions s'appliquent également aux énoncés complimenteurs de l'arabe tunisien. Mais pour cette dernière langue-culture, la réussite de cet acte (et de la louange de manière générale) tient aussi à la satisfaction d'une autre condition : suspecté d'attirer (volontairement ou non) le 'mauvais œil' sur son destinataire, le compliment doit se réaliser selon un mode qui neutralise en même temps la menace potentielle qu'il constitue intrinsèquement. Si le but d'un compliment est de faire plaisir à son destinataire, ce plaisir ne peut être obtenu que si ce compliment ne présente aucun risque d'atteinte du 'mauvais œil', sinon il se transformerait aussitôt en un acte menaçant. Comme nous allons le voir dans les paragraphes qui suivent.

\subsection{Les conditions d'emploi du compliment}

\section{La fréquence d'emploi}

La divergence dans la fréquence d'emploi du compliment entre le corpus arabe tunisien et le corpus français est frappante. En effet, un premier coup d'œil sur les deux corpus montre l'écart qui s'observe dans le nombre de compliments produits dans l'un et l'autre de nos deux corpus. Dans le corpus français, les compliments sont en effet, très fréquents ; pouvant apparaître dans des situations assez variées. Cette profusion est en outre confirmée dans d'autres travaux consacrés au compliment (Marandin, 1987[20] ; De Fornel,1989[7] ; Kerbrat-Orecchioni, 1994 [14]; Traverso, 1996 [27]). Comme l'ont souligné les auteurs de ces travaux, cet usage du compliment s'explique par le fait qu'en français celui-ci joue un rôle très important dans la relation. Il fonctionne en effet comme une routine de politesse positive et est très prisé et fortement attendu par les interactants : 
Si nous mettons en parallèle leur nombre et l'attente d'une évaluation qui existe chez chacun des interlocuteurs, il apparaît que tout se passe comme si chacun disait perpétuellement: «Qu'en penses-tu?», «Qu'en dis-tu?», «Est-ce-que c'est bien ? », et que chacun aussitôt répondait : «Oui, tu as bon goût », «Oui tu as l'air en forme », «Oui, tu agis de manière agréable ». Comme si sans cesse, les interactants quémandaient une évaluation positive, c'est-à-dire un mot qui les rassure. Cette remarque agréable, cette caresse verbale est nécessaire pour continuer à présenter de soi l'image attendue, et surtout indispensable pour continuer à avoir de soi cette image ». (Traverso, $1996: 110)$

En observant le corpus tunisien nous avons été frappée par la faible représentativité de cet acte de langage comparativement à ce qui se passe pour le français. Ce qui nous avait d'ailleurs amenée, pour les besoins de notre description, à recourir à d'autres types de données, en particulier à des exemples pris sur le vif.

Cet usage modéré de cet acte de langage s'explique sans doute, pour une grande part en tout cas, par le fait que, tout en étant un acte valorisant pour la face, le compliment est aussi largement ressenti comme étant empreint d'une menace potentielle, dans la mesure où il est susceptible d'attirer le 'mauvais œil'. Menace qui peut même entrainer leur proscription dans certaines circonstances : certains objets comme la santé, par exemple, sont en effet presque frappés de tabou.

En fait, lorsque chez l'interactant français l'attente d'une évaluation positive semble constamment sous - jacente, chez l'interactant tunisien tout se passe au contraire comme si chacun disait : «Ne t'occupe pas de moi », « Ne me regarde pas car ton regard peut porter l'envie ». Doutté (1909[6]) relevant cette censure qui pèse sur l'usage du compliment et de manière plus générale sur toutes formes de louanges chez les Nord- africains, écrivait déjà :

\begin{abstract}
[...] C'est bien pis quand l'envie s'exprime par des louanges : aussi nos indigènes craignent-ils les compliments, surtout quand ils viennent d'un inconnu, car il se peut qu'il ait le mauvais œil. C'est là sans doute qu'il faut voir l'origine d'un usage général dans l'Afrique du Nord : celui de donner à l'hôte l'objet qu'il a loué à haute voix ou dont il a montré qu'il avait envie » (Ibid. : 321).
\end{abstract}

Louis (1979 : 39 [18]) va dans le même sens lorsqu'il affirme que « le bédouin ne prise que fort peu l'admiration, ou du moins il ne la prise que modérément. Prodiguée de façon inopportune, elle attire « le mauvais œil » [...] et serait peut-être une forme cachée de la jalousie. Le peu d'admiration qu'il est licite d'extérioriser doit être soigneusement placé sous la protection divine [...] », et l'auteur d'ajouter :« Cette crainte du «mauvais œil» pousse le campagnard à souhaiter que le monde ne s'occupe pas de lui outre mesure et ne le montre pas du doigt [...] Facilement la disparition des qualités qu'il possédait ou qui ornaient ses enfants sera attribuée par le campagnard au trop d'empressement qu'apportèrent les autres à s'en occuper «tlâhet bîh en-nâs, les gens s'en sont trop occupés » (Ibid. : 40)

\title{
L'emplacement du compliment dans l'interaction
}

Dans sa description du compliment dans les conversations de visites, Traverso (1996 : 107$109[27])^{18}$ met en évidence le fait que cet acte de langage possède un emplacement privilégié au tout début d'une interaction, où il constitue une composante facultative, mais très fréquemment attestée, de la séquence d'ouverture. Cet emplacement privilégié est aussi signalé dans différentes études anglo-saxonnes ou françaises consacrées au compliment (op. cit). Il y est expliqué par le fait que les compliments, ayant rapport avec l'apparence, sont émis aussitôt que les participants se voient ; c'est-à-dire normalement au moment où 
l'objet du compliment est découvert. (Goffman, 1974 : 64-65 [8] associe de ce point de vue le compliment aux salutations).

Comme c'est généralement au tout début de la rencontre qu'ont lieu ces «découvertes», il n'est pas étonnant que les échanges complimenteurs se rencontrent surtout dans les séquences d'ouverture, dont ils constituent une composante facultative mais très fréquemment attestée. (Kerbrat-Orecchioni, 1994:217[14]).

Cet emplacement privilégié des compliments au tout début de la rencontre s'explique aussi par leur rôle : utilisés pour renforcer la solidarité et aussi pour rassurer les interactants sur l'image qu'ils ont et qu'ils donnent d'eux-mêmes, ils sont une expression directe de l'acceptation d'autrui. Ils jouent donc un rôle important au moment où les interactants se retrouvent.

Si le fait que le compliment soit produit au tout début d'une rencontre ou dès que l'objet est remarqué, semble aller de soi, en observant le corpus tunisien on constate que ce comportement n'est pas aussi naturel que cela puisse paraître. En effet, pour les Tunisiens, les premiers instants de l'interaction ne constituent point le moment le plus favorable pour produire un compliment, bien plus, on peut même dire que c'est un moment particulièrement défavorable. La plupart des personnes interrogées ont ainsi déclaré que lorsqu'ils remarquent un objet qui suscite l'admiration ou un changement chez l'interlocuteur et qu'ils décident de l'exprimer, ils ne le feront pas dès que l'objet en question est vu mais un peu plus tard après avoir abordé d'autres sujets. Il est donc tout à fait 'normal' qu'un compliment soit décalé par rapport au moment où l'objet est découvert, sans que cela ne nécessite une justification. Il semblerait en effet, que lorsque le compliment est produit au tout début d'une rencontre, il comporterait le risque d'être interprété comme l'expression d'une envie ou d'un sentiment jaloux. Et comme le compliment est souvent considéré comme porteur du 'mauvais œil', il y a là une autre raison de cette 'censure'. Le 'mauvais œil', en arabe «al aïn» (Litt. l'œil), est la malfaisance du regard envieux. Il est transmis par un regard chargé de haine ou d'envie ; mais il peut aussi être involontaire et résulter du pouvoir naturellement délétère d'un regard étrange et fixe. Or, justement, au moment où on découvre pour la première fois l'objet en question, notre regard, surpris, se dirige et se fixe naturellement -involontairement- vers l'objet ainsi remarqué. C'est ce regard-là qui est craint : c'est à ce moment-là que le regard est dit le plus « harcha » (Litt.« rude»), selon l'expression tunisienne, c'est à dire le plus 'mauvais'.

Ainsi, si la place privilégiée du compliment en ouverture s'explique en grande partie par la volonté des interactants d'inaugurer sous les meilleures auspices la relation interactionnelle, c'est aussi, paradoxalement, pour les mêmes raisons que le compliment est limité en ouverture. C'est que cet acte de langage n'a pas tout à fait les mêmes valeurs dans les deux sociétés concernées : acte essentiellement valorisant qui fonctionne comme une routine de courtoisie pour l'une, il est pour la seconde, quoique tout aussi valorisant, empreint en même temps d'une certaine menace.

\subsection{La valeur du compliment}

$\mathrm{Si}$, aujourd'hui, il est communément admis en pragmatique interactionnelle que les actes de langage se classent globalement (eu égard à l'effet qu'ils ont potentiellement sur la face et/ou le territoire des participants à l'interaction) en « actes intrinsèquement menaçants pour la face » (Face Threatening Acts : FTA) et « actes intrinsèquement valorisants pour la face »(Face Flattering Acts : FFA), on sait aussi depuis Kerbrat-Orecchioni (1992[13]et 1994 [14]) que la plupart des actes de langage ont un statut complexe en tant que FTA ou FFA. 
En effet, la plupart des actes accomplis au cours des interactions comportent simultanément les deux valeurs.

Cependant, comme l'ont montré différentes études comparatives, selon les sociétés la hiérarchie et le poids accordés à ces valeurs ainsi que les issues préférées pour sortir de « la double contrainte » dans laquelle nous plonge les actes « à double tranchant » (KerbratOrecchioni) varient parfois considérablement. C'est ce qui se passe en l'occurrence pour le compliment.

En français, comme s'accordent à l'affirmer les différents auteurs cités, il semble que la composante valorisante pour l'allocutaire (A) prédomine. La menace que peut constituer le compliment apparaît, elle, comme secondaire. Plusieurs indices vont dans le sens de cette interprétation :

- le compliment peut fonctionner comme une routine d'ouverture : formulé au début d'une rencontre (de visite pour le cas qui nous occupe) il est « une expression directe de l'acceptation d'autrui » (Traverso, 1996 : 108 [27]). La possibilité pour le compliment de jouer ce rôle tient donc entre autres à la valeur positive qui lui est accordée.

- il se prête volontiers à la formulation intensive : on sait qu'il existe un principe général selon lequel les actes menaçants ont tendance à être minimisés tandis que les actes valorisants sont hyperbolisés. ${ }^{19}$

- il répond à une forte attente de la part des interactants : s'il n'est pas obligatoire au sens propre du terme, il est tout de même très attendu dans certains contextes, à tel point que son absence, en créant une incertitude chez la personne qui l'attend, peut constituer une menace. $^{20}$

- il est très fréquent. Cette fréquence va de pair avec la forte attente dont il fait l'objet et indique une fois de plus l'importance que revêt cet acte de langage dans l'interaction.

Ainsi, dans la culture française, le compliment se présente donc comme un comportement rituel qui relève de la politesse positive (Brown \& Levinson, 1978 [4]et Kerbrat-Orecchioni, 1992, 1994[13,14]), c'est à dire qui vise avant tout à faire plaisir à l'autre, à confirmer le lien et à renforcer la solidarité.

Ce qu'on constate à l'étude du compliment en arabe tunisien ce sont les nombreuses contraintes qui pèsent sur sa réalisation. Contraintes qui touchent, nous l'avons vu, aussi bien à sa formulation qu'à son emploi et qui peuvent aller, dans certains contextes extrêmes, jusqu'à sa censure. Contraintes qui nous amènent à nous interroger sur la valeur de cet acte en tant que FFA ; valeur principale qu'on lui reconnaît ordinairement. En effet, les restrictions auxquelles se soumet le compliment et le travail de figuration («facework ») dont il fait l'objet sont plutôt ceux d'un acte menaçant. Mais est-ce à dire que le compliment est un FTA? Certainement pas. En fait, beaucoup plus qu'en français, le compliment en arabe tunisien se situe entre les deux et se présente comme un acte à double tranchant qui place les interactants dans un dilemme :

\section{Le compliment est un acte valorisant pour la face de l'allocutaire $A$}

Dans la mesure où le compliment porte un jugement positif sur l'allocutaire, il est forcément, pour ce dernier, un acte valorisant qui ne peut, au moins théoriquement, qu'être bien accueilli. Car, comme le souligne Kerbrat-Orecchioni, le besoin de voir flatter sa face positive est un fait universel :

[...] On ne peut pas concevoir de société dont les membres n'éprouvent pas le besoin de voir de temps en temps flatter leur face positive (1994:298 [14])

\section{Le compliment est un acte menaçant pour l'allocutaire $A$ :}




\section{Menace pour le territoire personnel}

Par le fait que le compliment est un jugement, une évaluation qui suppose que l'on ait observé son allocutaire, il est facilement perçu, dans la société tunisienne, comme un acte d'ingérence dans le territoire personnel du destinataire du compliment : ce dernier pouvant ainsi se sentir en quelque sorte épié. Ce caractère d'ingérence sera plus ou moins ressenti selon la nature de la relation entre les interlocuteurs (un compliment venant d'un inconnu aura plus tendance à être considéré comme tel) et selon la sensibilité de chacun (on peut être plus ou moins sensible à la protection de son territoire).

C'est sans aucun doute sur ce que Traverso (1996:111[27]) appelle « le commentaire de site » (un commentaire positif fait sur le lieu de la visite : maison, appartement...) que ce soupçon d'ingérence pèse le plus. Les remarques et même les louanges faites sur le cadre de vie de l'hôte peuvent en effet facilement être considérées par ce dernier comme une curiosité et être jugées comme déplacées et impolies. Dans les cultures arabes, le lieu de vie, le 'chez soi' est une « horma» c'est-à-dire, littéralement, une « inviolabilité », un lieu réservé et 'sacré' que le visiteur est tenu de respecter. Aussi, lorsque le Tunisien pénètre chez quelqu'un, on s'attend généralement à ce qu'il manifeste une certaine discrétion. Ce soupçon d'ingérence sera d'autant plus fort si la remarque ou le compliment sont produits au tout début de la rencontre. Pour empêcher que le commentaire de site et le compliment d'une manière générale soient empreints de menaces pour le territoire personnel du destinataire, certaines précautions sont donc nécessaires ; entre autres :

- s'assurer que la relation le permette. Dans ce cas, il faut non seulement que les participants soient des familiers ou des proches, mais il faut aussi s'assurer que l'allocutaire n'est pas en mesure d'interpréter l'acte en question comme une ingérence, ou encore de l'envie.

- il est nécessaire en outre, en tout cas plus prudent, de ne pas produire le compliment au tout début d'une rencontre, (surtout lorsque la relation est mal définie).

Mais cette menace pour le territoire personnel, quel que soit son degré, n'explique pas tout sur la forte contrainte qui pèse sur l'accomplissement de l'acte complimenteur et les nombreuses précautions qui entourent sa réalisation.

Menace symbolique pour l'individu : le 'mauvais œil'

Selon la croyance au 'mauvais œil', on considère que le désir ou l'admiration que l'on peut éprouver et même l'envie sont des sentiments incontrôlables dont on n'est pas toujours responsable. Partant de là, toute personne (qu'elle soit ou non prédisposée) est supposée capable de transmettre par son regard le 'mauvais œil', y compris d'ailleurs à soi-même. Dans ce sens, non seulement les compliments, mais aussi toutes autres formes de louange : celles qui se rapportent à un tiers par exemple et même le contentement qu'on éprouve pour soi, est susceptible d'attirer cette force maléfique.

Que risque 'la victime' du 'mauvais œil'?

Tout ce qui suscite l'admiration et attire pour cela le regard est exposé au 'mauvais œil'. Celui-ci peut se manifester de diverses manières :

- destruction ou perte de l'objet admiré.

- perte de la situation, de l'événement ou de l'objet du plaisir et du contentement. Un événement heureux (mariage etc.) ou une situation favorable (richesse, abondance des récoltes, pluie etc) peuvent ainsi se transformer en malheurs si elles sont trop mises à l'avant par les autres et même par soi-même. Une trop grande assurance, une trop grande satisfaction, ou des louanges jugées démesurées comme s'extasier devant l'abondance de la pluie ou des récoltes par exemple, se féliciter ou féliciter les autres pour une réussite, un gain ou tout autre événement peuvent, selon la croyance, entraîner le renversement de ces situations. 
- lorsque l'admiration se focalise sur l'individu (sur ses qualités physiques : beauté, bonne santé etc) c'est la personne même qui peut être en danger ; ce qui peut se traduire par la perte de ces qualités (par exemple par la maladie, voire la mort de cette personne).

Maladie et mort des individus, catastrophes en tous genres, destruction et perte des objets : les méfaits que l'on attribue au «al-aïn » (Litt. 'l'œil') sont innombrables. Le mauvais œil représente une cause, socialement admise et repérable, d'événements et de situations naturels : "C'est normal qu'il ne pleuve pas! A force d'avoir «crié » l'année dernière « Oh ! Quelle pluie, oh, il pleut beaucoup ! » sans jamais remercier Dieu !» est une des protestations qu'on entend très souvent.

Ainsi, tout en étant un FFA, le compliment se présente donc en même temps comme un acte intrinsèquement menaçant pour son destinataire. Rien d'étonnant donc à ce qu'il soit soumis, aussi bien dans sa formulation que dans son emploi, à tout un travail de figuration destiné à neutraliser les menaces qu'il suscite. Parmi ces procédés, nous avons pu relever : 1-Des procédés qui accompagnent la formulation du compliment et qui ont pour fonction d'annihiler la menace qu'il constitue intrinsèquement. Les plus fréquents étant

- l'emploi de formules conjuratoires : comme nous avons pu le voir, certaines formules conjuratoires accompagnent de façon presque systématique les énoncés louangeurs qu'ils soient focalisés sur A, sur L ou sur un tiers.

- le fait d'éviter une formulation intensive du compliment par des exclamations d'admiration (interjection, mimiques, adverbes d'intensité) lorsque l'objet de l'admiration est jugé vulnérable : une bonne santé, un enfant en bas âge, une femme enceinte...

- la substitution lexicale : dans certains cas, la formule conjuratoire se substitue au terme axiologique pour exprimer une évaluation positive.

2- Des procédés qui règlent la production du compliment.

En arabe tunisien, certaines contraintes pèsent sur la production du compliment. Elles concernent en particulier :

- la nature de la relation interpersonnelle devant exister entre le complimenteur et le complimenté. Comme pour le français, le compliment manifeste une prédilection pour la relation familière, mais il faut aussi que la relation permette aux interlocuteurs de se sentir en confiance.

- l'objet sur lequel porte le compliment: pour qu'un compliment réussisse, c'est-à-dire qu'il puisse faire plaisir à son destinataire, il faut savoir que certains objets et certaines situations se prêtent mal aux compliments : par exemple certains aspects de l'apparence physique (ex les cheveux longs), la santé, les enfants en bas âge, ou encore la situation d'une femme enceinte ou célébrant un mariage.

- l'emplacement du compliment: nous avons montré plus haut que pour réduire ou neutraliser la menace du 'mauvais œil' inhérente à toute expression d'admiration, il convient d'éviter de produire un compliment au tout début d'une rencontre c'est-à-dire dès que l'objet est remarqué.

\section{Conclusion}

Le but de notre comparaison $\mathrm{du}$ fonctionnement du compliment dans les deux langues/cultures, française et tunisienne, était de mettre en évidence les variations significatives qui l'affectent. Significatives en ce qu'elles nous renseignent sur la conception que chacune de ces deux langues se fait de cet acte. Ainsi, et malgré les limites de notre description, nous avons pu observer que si en français le compliment fonctionne principalement comme un acte valorisant et une routine de courtoisie faisant partie de la politesse positive, il se présente en arabe tunisien comme un acte beaucoup plus complexe dans la mesure où la valeur menaçante - associée à la croyance au 'mauvais œil'- s'impose au même degré que sa valeur en tant que qu'acte valorisant pour la face positive. Bien plus, 
dans certains contextes, c'est même l'aspect menaçant qui s'imposera. Contrairement à ce qui se passe pour le français, dans la société tunisienne, lorsque les interlocuteurs se trouvent pris dans un dilemme (entre composante valorisante et composante menaçante), ils auront tendance à le résoudre par le silence.

\section{Références}

1. A. Auchlin, Faire, montrer, dire. Pragmatique comparée de l'énonciation en français et en chinois, Peter Lang, Berne (1993)

2. C. Beal, Les interactions verbales interculturelles: quels corpus? Quelle méthodologie?, In Traverso, V. (éd.), Perspectives interculturelles sur l'interaction, PUL, Lyon, 13-32 (2000)

3. S. Blum-Kulka, J. House \& G. Kasper (Eds.), Cross-Cultural Pragmatics: Requests and Apologies. N.J. Norwood, Ablex (1989)

4. P. Brown, S. Levinson, Universals in human usage: politeness phenomena, In E.Goody (Eds.), 56-290 (1978)

5. P. Dornier, La politesse tunisienne, IBLA, 39, 33 (1952)

6. E. Doutté, Magie \&Religion dans l'Afrique du Nord, Typographie Adolphe Jourdan Imprimeur- Libraire -Editeur, Alger (1909)

7. M. De Fornel, Actes de langage et théorie du prototype : l'exemple du compliment, Cahiers de praxématique, $12: 37-49$ (1989)

8. E. Goffman, Les rites d'interaction, Minuit, Paris (1974)

9. J.J. Gumperz, Discourse strategies, CUP, Cambridge (1982), trad. Chap. 1-5 et 8-9: Sociolinguistique interactionnelle, L'Harmattan, Paris (1989)

10. J. Holmes, Paying compliments : a sex-preferential politeness strategy, Journal of Pragmatics, 12/4, 445-467 (1988)

11. L. Kastler, La politesse linguistique dans la communication quotidienne en français et en russe, Thèse de doctorat de l'Université Lumière Lyon 2 (1998)

12. S. Katsiki, Les actes de langage dans une perspective interculturelle : l'exemple du vœu en français et en grec, Thèse de doctorat de l'Université Lumière Lyon 2 (2001)

13. C. Kerbrat - Orecchioni, Les interactions verbales, vol. II, Paris: A. Colin (1992)

14. C. Kerbrat - Orecchioni, Les interactions verbales, vol. III, Paris: A. Colin (1994)

15. C. Kerbrat - Orecchioni, Les actes de langage dans une perspective interculturelle: problèmes théoriques et descriptifs, In V.Traverso (Eds.), Perspectives interculturelles sur l'interaction, PUL, Lyon75-92 (2000)

16. C. Kerbrat - Orecchioni, Les actes de langage dans le discours. Théorie et fonctionnement, Nathan Université, Paris (2001)

17. C. Kerbrat - Orecchioni, Le discours en interaction, A. Colin, Paris (2005)

18. A. Louis, Dans le sud de la Tunisie, du nomadisme à la sédentarisation. Echanges, Vol.1. N¹, 69-84 (1979)

19. G. Leech, Principles of Pragmatics, Longman, Londres / New York (1983)

20. J.M. Marandin, Des mots et des actions. Compliment, complimenter et l'action de complimenter. Lexique, 5, 65-89 (1987)

21. W. Olesky (Eds.), Contrastive Pragmatics, John Benjamins,Amesterdam/Philadelphia, (1989)

22. A. Piamenta, Islam in Everyday Arabic Speech, Leyde, Brill (1979)

23. A. Piamenta, The Muslim Conception of God and Human Welfare as reflected in Everyday Arabic Speech, Leyde, Brill (1983)

24. J. Searle, Les actes de langage, Hermann, Paris (1972)

25. J. Searle, Sens et expression, Minuit, Paris (1982)

26. V. Traverso, La conversation familière. Les interactions verbales dans les visites, Thèse de l'Université Lumière Lyon 2 (1993) 
27. V. Traverso, La conversation familière, PUL, Lyon (1996)

28. V. Traverso, Autour de la mise en ceuvre d'une comparaison interculturelle, In V. Traverso (Eds.), Perspectives interculturelles sur l'interaction, PUL, Lyon 33-51 (2000)

29. V. Traverso (Eds.), Perspectives interculturelles sur l'interaction, PUL, Lyon (2000)

30. V. Traverso, Des échanges ordinaires à Damas: aspects de l'interaction en arabe, PUL, Lyon (2006)

31. V. Traverso, Repères pour la comparaison d'interactions dans une perspective interculturelle, Les carnets du CEDISCOR, 9|2006, mis en ligne le 07 juin 2010, disponible sur : http://cediscor.revues.org/617 (2006)

32. J.Verschueren, The semantics of forgotten routines, In F. Coulmas (Eds.), Conversational Routine, Mouton, La Haye, Paris, New York,133-153 (1981)

33. A.Wierzbicka, A semantic metalangage for cross-cultural comparison of speech acts and speech genres, Language and society, 14, 491 - 514 (1985)

34. A. Wierzbicka, Cross-cultural pragmatics. The semantics of human interaction, Mouton de Gruyter, Berlin/New York: (1991)

35. N. Wolfson, Compliments in Cross-Cultural Perspective, Tesol Quarterly 15, 2, 117 $124(1981)$

\section{Convention de transcription}

Litt. traduction littérale de l'arabe.

( ) utilisés pour encadrer des mots français employés dans le corpus

، sont utilisés pour encadrer les expressions traduites littéralement (essentiellement les formules)

/ intonation montante (dans le corpus arabe elle exprime la surprise ou l'admiration)

intonation descendante

[ ] encadrent des passages non pris en compte dans la description pause

(Dans la transcription du corpus arabe nous avons séparé par un (-) les morphèmes de personnes, sujet (y-) ou complément (-ik) par exemple, de l'article...

${ }^{1}$ L'ouvrage en question est issu de sa thèse intitulée La conversation familière. Les interactions verbales dans les visites, soutenue à l'Université Lumière Lyon 2 (1993a) 


\begin{abstract}
${ }^{2}$ Nous avons pu, grâce à la générosité de l'auteure, avoir accès à ce corpus
${ }^{3}$ La constitution du corpus arabe est antérieure à notre réflexion sur le compliment : à l'époque où nous avions effectué les enregistrements nous n'avions encore aucune hypothèse concernant les variations observées dans le fonctionnement du compliment

${ }^{4}$ Voir pour les questions de transcription et de traductions des corpus arabes Traverso (2006 : 79-98)

${ }^{5}$ Nous ne pouvons, dans le cadre limité de cette étude, aborder toutes les questions ayant trait aux problèmes méthodologiques liés à l'approche contrastive sur corpus. Nous renvoyons pour cela entre autres à Béal (2000), Traverso (2006), Kerbrat-Orecchioni (2005)
\end{abstract}

${ }^{6}$ Voir Kerbrat-Orecchioni (1994) sur les problèmes posés par l'identification du compliment : problème de ce qu'il faut entendre par évaluation positive (dans la mesure où la valeur axiologique d'un terme peut varier en fonction des systèmes évaluatifs propres aux locuteurs et aux cultures), problèmes de la formulation implicite, problème de «la chaîne de mérite », c'est-à-dire, de cet ensemble d' individus et d'objets plus ou moins fortement associés à l'allocutaire et dont celui-ci se sent solidaire (cette chaîne de mérite étant, comme le montre l'auteure, variable d'un individu à un autre et surtout d'une culture à une autre.).Problèmes qui amènent l'auteure à conclure que « la valeur complimenteuse d'un compliment doit bien souvent être négociée dans et par l'interaction grâce à des procédés implicites ou explicites [...] » $(1994: 221)$

${ }^{7}$ Selon que le compliment porte directement sur l'allocutaire A ou, indirectement, sur une personne, différente de A mais qui lui est associée, Kerbrat-Orecchioni (1994) distinguera les «compliments directs» et les «compliments indirects ». Mais pour qu'une intervention puisse être interprétée comme un compliment indirect, il faut que la relation qui lie l'allocutaire A à la personne concernée par l'éloge soit suffisamment étroite pour que le compliment puisse par ricochet affecter A. C'est, par exemple, le cas ( le plus évident) d'une relation parent-enfant. Mais hormis ce cas, le plus évident, se pose la question de savoir quels sont les types de relation («étroites») qui permettent à un compliment porté sur un tiers de ricocher sur A. Il est clair en tout cas que le type de relation varie selon les individus et selon les cultures

${ }^{8}$ Mais pour ces deux verbes le sens de la louange ne constitue qu'une des significations possibles

${ }^{9}$ Ce serait confondre 'acte de langage', qui est une catégorie abstraite, (des « espèces conceptuelles » selon Searle (1982:34) et verbe ou lexique désignant ce concept. Or on sait aujourd'hui que les actes illocutoires ne doivent pas être identifiés au verbes illocutoires et que la liste des actes de langage ne coïncide pas avec celle des termes qui les décrivent dans une langue donnée ( il s'agit là d'un problème complexe qui est examiné entre autres dans Searle (1982), et Kerbrat-Orecchioni, 2001).

${ }^{10}$ A distinguer des compliments « indirects » qui concernent une personne à laquelle l'interlocuteur est lié de façon plus ou moins forte (enfants, conjoints etc.)

${ }^{11}$ Mais la valeur axiologique d'un terme peut varier en fonction des systèmes évaluatifs propres aux énonciateurs et donc dépendre du contexte.

${ }^{12} \mathrm{Au}$ sujet de l'emploi de cette formule, Piamenta (1979: 88) fait remarquer que «In everyday language, however, short variations of the tas'liya (...) are used in talks and tales, when wishing to avert the evil eye, when expressing admiration or satisfaction, when calling for help, asking for patience, conjuring, swearing, complying with someone's wish, blessing, or straining one's memory in order to recall something or the location or a lost object ».

${ }^{13}$ La référence religieuse présente dans un grand nombre de formules pose la question de savoir s'il existe chez les locuteurs une conscience permanente de la référence à Dieu ou si elle s'est effacée. Nous pensons que la plupart des formules sont aujourd'hui figées et désémantisées, mais aussi que la valeur religieuse 'latente' peut se réactiver.

${ }^{14}$ Comme c'est le cas pour la plupart des formules dites de bénédiction, l'intervention initiative appelle souvent une réaction sous forme d'un retour de vœu formulé à partir de la même base morpho-lexicale. 
${ }^{15}$ Pour plus de détail au sujet de la réaction au compliment, nous renvoyons à Kerbrat-Orecchioni (1994: 230-268)

${ }^{16}$ Comme nous l'avions expliqué plus haut, l'étude de la valeur du compliment en arabe nous a amenée à prendre en compte les différents types d'actes exprimant une évaluation positive

${ }^{17}$ Le poisson, comme d'autres objets, étant considéré comme un prophylactique, capable d'éloigner le mauvais œil.

${ }^{18}$ Voir aussi Kerbrat-Oreccioni (1994 : 216-218).

19 «Les preuves sont nombreuses de l'existence d'un principe voulant que, d'une manière générale, on adoucisse les FTAs, et qu'on renforce les anti-FTAs ; qu'on litotise les comportements impolis, et qu'on hyperbolise les comportements polis, conformément à ce qu'énonce Leech (1983: 146) : «There will be a preference for overstating polite beliefs and for understating impolite ones » $[\ldots]$ (Kerbrat-Orecchioni, $1992: 228$ ).

${ }^{20}$ Constatant la présence massive des compliments dans les conversations familières, Traverso a été amenée à conclure : «Il semble exact que nous soyons toujours en attente d'une évaluation positive de nos faits et gestes, qui nous rassure [...] si on le soupçonne si facilement d'insincérité, c'est qu'au fond, on y attache une véritable importance, qu'on le réclame, le quémande, l'attend et que son absence introduit un doute $\gg(1996: 270-273)$. 